\title{
Comparative Study between two Types of Embryo Transfer Catheters: A Randomized Controlled Trial
}

\author{
Original \\ Article Hassan Awad Bayoumi, Mostafa Fouad Gomaa, Gihan El-Sayed El-Hawwary, \\ Ahmed Abdel Shafy El-Shahawy \& Mohab Ahmed Fouad El-Rabat
}

Department of Obstetrics and Gynecology Department, Faculty of Medicine, Ain Shams University

\begin{abstract}
Background: Embryo transfer is a crucial step in IVF-embryo transfer treatment cycles, but it is probably the most inefficient. Ovarian stimulation, oocytes retrieval, fertilization and embryo culture have been extensively studied and are performed under tight control, while improvements in embryo transfer are less often considered. There are many factors, in addition to the embryo quality, that have been shown to influence the success of embryo transfer such as the technique used, the experience of the operator and the difficulty of the procedure.

Aim of the Work: To compare the efficacy of the embryo transfer catheters: Wallace ${ }^{\circledR}$ and Prince ${ }^{\circledR}$ in an IVF program of a tertiary referral university center.

Patients and Methods: A randomized controlled research trial conducted at private IVF unit and the unit of Reproductive Medicine of Ain-Shams University. Study subjects have been recruited from an IVF management cycle. The research study has been conducted from January 2016 till September 2019.

Results: The current research study investigated four types of embryonic catheters Wallace, Prince with and without mandrel and Labotech most of the research study findings did not reveal any statistical significant differences by statistical analysis in which basic features and ovarian stimulation features did not differ between research groups categorized according to the type of catheter implemented.

Conclusion: Another issue that should be considered in future research is the integration of uterine volume, cervical position, uterine axis as factors affecting the choice of the type of catheter that could aid in proper performance of this meticulous art of embryonic transfer besides differences in experience and practice performed by the reproductive clinician should be put in consideration. Future research studies should consider multicentric fashion of research with great consideration of age, BMI, ethnicity, infections (causing cervical edema) as factors affecting the anatomic integrity and position therefore affecting the ease of the embryonic transfer process.
\end{abstract}

Key Words: Embryo transfer catheters, dimished ovarian reserve, ovarian dysfunction

Received: 18 July 2019, Accepted: 24 August 2019

Corresponding Author: Mohab Ahmed Fouad El-Rabat, MD, Department of Obstetrics and Gynecology, Al Galaa Maternity Teaching hospital, Cairo, Egypt, Tel.: +201016106423, E-mail: ramadagroupegypt@gmail.com

ISSN: 2090-7265, August 2019, Vol. 9, No. 3

\section{INTRODUCTION}

Several studies have shown increases in clinical pregnancy rate $(\mathrm{PR})$ resulting from improvements in the various aspects of embryo transfer including cervical cleaning, a full bladder, dummy transfer, pretreatment cervical dilatation in difficult cases and ultrasound guidance $^{[1]}$.

It has also been suggested that catheter choice influences pregnancy rates ${ }^{[2]}$ and several surveys in the UK and Australia have shown that embryo transfer catheters rank high as an important independent factor in the success of an IVF program ${ }^{[3]}$.

A systematic review has suggested that the ideal embryo transfer catheter should avoid any trauma to the endocervix and/or the endometrium as it is introduced into the uterine cavity ${ }^{[3]}$.

It was, therefore, decided to evaluate the performance of the Wallace, Labotech and Prince Catheters in a prospective randomized controlled trial (RCT) using clinical PR as the main outcome measure in continuing efforts to determine the optimal embryo transfer device.

Embryo transfer can lead to the success or failure of an assisted reproductive technology (ART) cycle $^{[5]}$.

One factor in ET, that has been identified as a variable that can positively or negatively affect pregnancy rate (PR), is the type of transfer catheter used ${ }^{[2]}$. It has been reported that more flexible catheters, such as the EdwardsWallace, resulted in significantly higher PRs than the more rigid Frydman Tight Difficult Transfer (TDT) (Fertility Technologies, Natick, MA) catheters ${ }^{[4]}$. In 2001, Boone et al. ${ }^{[6]}$ demonstrated a trend toward improved PRs with the Edwards- Wallace (Cooper Surgical, Shelton, CT) catheter compared with the Cook Soft-Pass (Cook Urological, Spencer, IN) catheter ${ }^{[6]}$. 


\section{AIM OF THE WORK}

To compare the efficacy of the embryo transfer catheters: Wallace ${ }^{\circledR}$ and Prince ${ }^{\circledR}$ in an IVF program of a tertiary referral university center.

\section{PATIENTS AND METHODS}

A randomized controlled research trial conducted at private IVF unit and the unit of Reproductive Medicine of Ain-Shams University. Study subjects have been recruited from an IVF management cycle.

The research study has been conducted from January 2016 till September 2019.

\section{Inclusive research criteria}

- Cases under $<40$ years of age

- $\quad$ Cases having a Body mass index (BMI) from 18-29 $\mathrm{kg} / \mathrm{m}^{2}$.

- Cases had at least two embryos developed till day 5 obtained from a fresh IVF cycle

- ART cycle (IVF/ICSI) used fresh sperm and oocytes

- First IVF/ICSI management cycle.

\section{Exclusive research criteria}

- Patients who have had single embryo transfers

- Cases aged are 40 years and above.

- Cases with a Body mass index (BMI) below $18 \mathrm{~kg} /$ $\mathrm{m}^{2}$

- Cases performing IVF management in conjunction with pre implantation genetic diagnosis or previous $\mathrm{t}$ chemo/radiotherapy.

- Cases with repeated IVF failures ( $>3$ trials) or a prior difficult embryonic transfer.

- Severe oligo/terato/atheno spermia syndrome.

- Endometriosis severity above stage II

- Poor responders;

- Low serum estradiol (E2) levels (500pg/ $\mathrm{ml}$ or less) assayed on the day of human chorionic gonadotropin (HCG) trigger

- Less than four oocytes retrieved on the day of oocyte retrieval.

Suspected poor responders according to the European Society of Human Reproduction and Embryology (ESHRE) at least two of the following three criteria had to be present to establish the definition:

1. Advanced maternal age (above 40 years) or any other risk factor for poor ovarian response.

2. A previous poor ovarian response (less than 3 oocytes with a conventional stimulation protocol).
3. An abnormal, ovarian reserve test [i.e. antral follicle count (AFC) less than 5-7 follicles or anti-Mullerian hormone (AMH) below $0.5-1.1 \mathrm{ng} / \mathrm{ml}$.

Cases fulfilling the research inclusive and exclusive research criteria and after informed written consent the recruited cases have been randomized by usage of sealed opaque envelope method into one of two research groups:

- Research Group I: Cases had embryonic transfer undergone by usage of PM TRANS SET Catheter without mandrel.

- Research Group II: Wallace research group.

\section{Research study Intervention:}

All the cases prepared for embryo transfer have undergone the following: careful and detailed-clinical history to reveal the cause of infertility.

\section{Personal history:}

Name, age, occupation, residence and special habits of medical importance.

\section{A. Menstrual history:}

First day of last menstrual period, type of infertility and previous history of ART.

\section{B. Past history}

History of any medical disorder or surgical history.

\section{Husband history}

\section{Examination of the cases:}

Proper examinations have been conducted to reveal the cause of infertility following the proper steps:
a. General examination
b. Abdominal examination
c. Vaginal examination
d. Sonographic examination.

Induction of ovulation has been performed to all study subjects as follows:

- On day 3 of spontaneous cycles, all cases had basal hormonal profile assay (FSH, LH, E2, TSH and prolactin) for ovarian reserve screening.

- Transvaginal sonography performed on day 3 of non-stimulated cycles conducted by usage of transvaginal probe of 5-9 MHZ. Any case discovered to have uterine or tubal pathology has been excluded.

- Controlled Ovarian hyperstimulation protocol has been performed as follows: long GnRH agonist protocol starting from mid-luteal. Phase by daily subcutaneous injection of triptoreline acetate (Decapeptyl $0.05 \mathrm{mg}$, Ferring Pharmaceutical, Kid, Germany). Then on day 3 of next cycle, ovarian 
hyperstimulation has been initiated by daily injection of HMGr (Menogon 75 lU/amp. "Ferring Pharmaceutical, Kid, Germany or Merional 75 lU/ amp" IBSA, Switzerland"). The starting dose of gonadotropins has been prescribed according to the age and body built of the study subjects, then the dosage was accustomed. According to the ovarian responsiveness that has been assessed by transvaginal folliculometry performed on day six of the cycle.

- According to the ovarian responsiveness, day after day trans vaginal sonography has been conducted and at the moment when the leading follicle reaches $16 \mathrm{~mm}$, daily trans vaginal sonography has been performed till the largest follicle reaches a diameter of $>18 \mathrm{~mm}$. The maximum duration of $\mathrm{HMG}$ will not be allowed to exceed day 16 .

- HCG (Choriomon 10,000 IU/amp. "IBSA, .Switzerland") has been administered for triggering ovulation.

\section{Ovum pick up:}

- 36 hours after HCG injection, the transducer has been connected to the ultrasound system. The direction of the guide beam has been checked. The puncturing needle was connected to an aspiration apparatus attached by a Fixation ring to the front and rear ends of the vaginal transducer, thereby defining the direction of puncture corresponding to the guide beam on the ultrasound image.

- The aspiration has been checked using test tubes. The uterus, both ovaries and iliac vessels, will be identified by the visualization in both plans. The distance between the upper pole of the vagina and the ovary was closed and evaluated (care was taken to prevent intestinal or vascular interposition). Depth localization of the closest accessible follicle (distance from the upper vaginal pole to the center of the follicle) will be done. Needle will be pushed forcefully to the center of the follicle (Aspiration pressure 90-100 $\mathrm{mmHg}$ ).

\section{$I V F \sim I C S T:$}

- Intracytoplasmic sperm injection was performed on metaphase II oocytes using the direct penetration technique. Fertilization results will be assessed 16 to 19 hours after ICSI. Fertilization was considered normal by the presence of two pronuclei. Oocyte degeneration was identified by collapse of cytoplasmic contents and separation from the zona. Failed fertilization was defined by the absence of the pronuclei.

\section{Embryo transfer:}

All patients had mock embryonic transfers performed prior to their IVF cycle as this is routine practice in the study unit. On the day of actual embryo transfer, 300 patients were randomly allocated to the Wallace group, 300 to the Prince group. For embryo transfer, to eliminate any of the reported variations in success rates due to a (physician factor), a single physician performed all ETs, All ETs were performed on day 5 after retrieval and an identical technique was used in all patients that placed supine in the lithotomy position. The cervix was exposed using a Cusco bi-valve speculum. Any cervical mucus encountered was gently removed with a simple swab soaked in phosphate-buffered saline.

Cases had a full bladder for embryo transfer and the uterus will be visualized using ultrasound with an abdominal probe. Under ultrasound control, the catheter was introduced into the mid-uterine cavity taking care to avoid touching the uterine fundus. The embryos were then gently expelled with the aid of a tuberculin syringe. The catheter remained in place for 20-30s after the embryos were expelled. The transfer catheter was then flushed with medium under light microscopy to ensure that no embryos will be retained in the catheter. Experienced competent operator will perform all transfers having performed a minimum of 200 transfers independently prior to the commencement of the study and familiar with the two catheters investigated in this study. Following embryo transfer, patients were kept in bed for 2030 min before discharge. We classified all embryo transfer procedures as smooth procedures that occur without the usage of any force or other form of instrumentation with no requirement to change the catheter. Luteal phase support was given in the form of progesterone pessaries (Cyclogest; Hoechst, Frankfurt, Germany). Serum HCG concentration was measured 14 days after oocyte retrieval and if positive, an ultrasound was performed 14 days later, i.e. at 6 weeks gestation.

Details describing the number of embryos available and the number transferred, the grade of the embryo transfer, whether any embryos remained in the catheter after transfer, whether a change of catheter was necessary and the visibility of the catheter under ultrasound was recorded. Additionally, the patient was asked to score any discomfort during the transfer using a visual linear analogue score.

\section{Defining pregnancy}

Biochemical pregnancy was confirmed by positive pregnancy test performed 12 days after embryonic transfer and repeated after $48 \mathrm{~h}$.

Clinical pregnancy was defined by the existence of a gestational sac by means of transvaginal sonography performed 6 weeks after embryonic transfer.

\section{Embryo transfer catheters}

The Prince PM 'TRANS SET Embryo transfer catheter (Prince Medical, France) consists of one graduated sheath of introduction in polyprolene with $1 \mathrm{~cm}$ markings to indicate depth of penetration into the endocervix (in the inside of which is: positioned a mandrel that can be performed). The inner catheter is made of soft polyethylene with a hot-formed tip profile to minimize endocervical trauma; the outer and inner diameters of the inner transfer catheter are 1.5 and 2: $\mathrm{mm}$, respectively (Prince Product information leaflet). 
The Wallace catheter (Sims Portex Ltd, Hythe, UK), a soft embryo transfer catheter, also has a flexible marked outer sheath to indicate depth of penetration and a soft inner catheter which is made from a non-toxic biocompatible medical polymer. The outer and inner diameters of the Wallace inner catheter are 1.6 and $0.75 \mathrm{~mm}$, respectively (Wallace Product information leaflet).

All research data obtained have been documented on preformed case record form.

\section{Randomization:}

Recruited cases have been randomized into one of the research study groups. Randomization has been performed by usage of a computer-generated randomization system.

\section{Allocation:}

- Was performed by using dark sealed envelopes containing the intervention derived from computer generated list were created by a third party not involved in the allocation process then randomization was performed by picking one envelope for each case from sequenced number envelopes by a nurse not involved in the study.

\section{Statistical Methods}

Statistical analysis has been performed by usage of Statistical Package for Social Sciences (SPSS $®$ ) for Windows' version 16.0. Research data have been expressed in the form of range, mean and standard deviation (for numeric parametric research variables), range, median and interquartile range (for numeric non-parametric research variables) and number and percentage (for categorical variables). Difference between variables of two research groups was analyzed by implementing independent student's t-test (for numeric parametric variables), MannWhitney's [/-test (for numeric non-parametric variables) and continuity-corrected Chi-squared or Fischer's Exact (for categorical variables). Association between two variables was assessed implementing Pearson's correlation coefficient (for parametric research variables) and
Spearman's rank correlation coefficient (for non-parametric research variables).

\section{RESULTS}

Comparison between Wallace and Prince (without mandrel)

Table 1 shows that no significant difference between Wallace and Prince (without mandrel) groups regarding basal characteristics.

Table 2 shows that no significant difference between Wallace and Prince (without mandrel) groups regarding stimulation and fertilization.

Table 1: Basal characteristics among Wallace and Prince (without mandrel) groups

\begin{tabular}{|c|c|c|c|c|}
\hline Variables & Measures & $\begin{array}{l}\text { Wallace } \\
(\mathrm{N}=300)\end{array}$ & $\begin{array}{c}\text { Prince } \\
(\mathrm{N}=300)\end{array}$ & $\mathrm{P}$ \\
\hline \multirow{2}{*}{ Age (years) } & Mean \pm SD & $31.1 \pm 3.0$ & $31.2 \pm 2.9$ & \multirow{2}{*}{${ }^{\wedge} 0.668$} \\
\hline & Range & $23.0-38.0$ & $23.0-39.0$ & \\
\hline \multirow{2}{*}{ BMI (kg/m2) } & Mean \pm SD & $24.1 \pm 1.8$ & $24.0 \pm 1.9$ & \multirow{2}{*}{$\wedge^{\wedge} 0.754$} \\
\hline & Range & $18.8-28.4$ & $18.7-28.6$ & \\
\hline \multirow{2}{*}{ FSH (IU/L) } & Mean \pm SD & $7.08 \pm 0.89$ & $7.13 \pm 0.91$ & \multirow{2}{*}{${ }^{\wedge} 0.482$} \\
\hline & Range & $4.70-9.80$ & $4.80-9.90$ & \\
\hline \multirow{2}{*}{ LH (IU/L) } & Mean \pm SD & $5.66 \pm 1.08$ & $5.72 \pm 1.10$ & \multirow{2}{*}{${ }^{\wedge} 0.504$} \\
\hline & Range & $2.10-9.00$ & $2.60-9.20$ & \\
\hline \multirow{2}{*}{$\mathrm{E} 2(\mathrm{pg} / \mathrm{mL})$} & Mean \pm SD & $56.7 \pm 4.6$ & $56.7 \pm 5.2$ & \multirow{2}{*}{$\wedge^{\wedge} 0.988$} \\
\hline & Range & $43.4-70.0$ & $42.9-74.0$ & \\
\hline \multirow{2}{*}{$\begin{array}{l}\text { Progesterone } \\
(\mathrm{ng} / \mathrm{mL})\end{array}$} & Mean \pm SD & $9.23 \pm 1.78$ & $9.24 \pm 1.86$ & \multirow{2}{*}{$\wedge^{\wedge} 0.961$} \\
\hline & Range & $5.30-14.20$ & $5.20-13.70$ & \\
\hline \multirow{6}{*}{ Etiology } & Tubal & $91(30.3 \%)$ & $86(28.7 \%)$ & \multirow{6}{*}{$\# 0.117$} \\
\hline & Male & $84(28.0 \%)$ & $91(30.3 \%)$ & \\
\hline & DOR & $41(13.7 \%)$ & $52(17.3 \%)$ & \\
\hline & OD & $29(9.7 \%)$ & $24(8.0 \%)$ & \\
\hline & Unexplained & $50(16.7 \%)$ & $34(11.30 \%)$ & \\
\hline & Endometriosis & $5(1.7 \%)$ & $13(4.3 \%)$ & \\
\hline
\end{tabular}

DOR: Dimished ovarian reserve, OD: Ovarian dysfunction, ${ }^{\wedge}$ Independent t-test, \#Chi square test

Table 2: Stimulation and fertilization among Wallace and Prince (without mandrel) groups

\begin{tabular}{|c|c|c|c|c|}
\hline Variables & Measures & Wallace $(\mathrm{N}=300)$ & Prince $(\mathrm{N}=300)$ & $\mathrm{P}$ \\
\hline \multirow{2}{*}{$\begin{array}{l}\text { Total } \\
\text { gonadotropins (IU) }\end{array}$} & Mean \pm SD & $2032.0 \pm 371.6$ & $2051.5 \pm 396.5$ & \multirow{2}{*}{$\wedge 0.535$} \\
\hline & Range & $1275.0-3075.0$ & $1275.0-3075.0$ & \\
\hline \multirow{2}{*}{ Duration of stimulation (days) } & Mean \pm SD & $12.2 \pm 2.3$ & $12.3 \pm 2.3$ & \multirow{2}{*}{$\wedge 0.645$} \\
\hline & Range & $8.0-19.0$ & $8.0-19.0$ & \\
\hline \multirow{2}{*}{ Oocytes retrieved } & Mean \pm SD & $8.5 \pm 3.0$ & $8.3 \pm 3.0$ & \multirow{2}{*}{$\wedge 0.269$} \\
\hline & Range & $2.0-18.0$ & $2.0-18.0$ & \\
\hline \multirow{2}{*}{ Oocytes injected } & Mean \pm SD & $6.3 \pm 2.3$ & $6.4 \pm 2.2$ & \multirow{2}{*}{$\wedge 0.550$} \\
\hline & Range & $2.0-10.0$ & $2.0-10.0$ & \\
\hline \multirow{2}{*}{ Fetilized oocytes } & Mean \pm SD & $5.4 \pm 2.2$ & $5.5 \pm 2.0$ & \multirow{2}{*}{$\wedge 0.582$} \\
\hline & Range & $2.0-9.0$ & $2.0-9.0$ & \\
\hline \multicolumn{2}{|c|}{ Fertilization rate $(=$ Fertilized/injected $)$} & $86.3 \% 1633 / 1893$ & $86.2 \% 1661 / 1926$ & $\# 0.983$ \\
\hline \multirow{2}{*}{ Embryo good quality } & Mean \pm SD & $3.03 \pm 1.07$ & $3.07 \pm 1.02$ & \multirow{2}{*}{$\wedge^{\wedge} 0.612$} \\
\hline & Range & $1.00-5.00$ & $1.00-5.00$ & \\
\hline
\end{tabular}

${ }^{\wedge}$ Independent t-test, \#Chi square test 
Table 3 shows that no significant difference between Wallace and Prince (without mandrel) groups regarding transfer difficulty and patients' discomfort.

Table 3: Transfer difficulty and patients' discomfort among Wallace and Prince (without mandrel) groups

\begin{tabular}{lccc}
\hline Variables & Wallace $(\mathrm{N}=300)$ & Prince $(\mathrm{N}=300)$ & $\mathrm{P}$ \\
\hline Difficult transfer & $28(9.3 \%)$ & $23(7.7 \%)$ & $\# 0.464$ \\
Patients' discomfort & $23(7.7 \%)$ & $31(10.3 \%)$ & $\# 0.254$ \\
\hline
\end{tabular}

\#Chi square test

Table 4 shows that no significant difference between Wallace and Prince (without mandrel) groups regarding transfer characteristics.
Table 4: Transfer characteristics among successes Wallace and Prince (without mandrel) groups

\begin{tabular}{llccc}
\hline Variables & Measures & $\begin{array}{c}\text { Wallace } \\
(\mathrm{N}=277)\end{array}$ & $\begin{array}{c}\text { Prince } \\
(\mathrm{N}=269)\end{array}$ & $\mathrm{P}$ \\
\hline \multirow{2}{*}{ Loaded embryos } & Mean $\pm \mathrm{SD}$ & $2.63 \pm 0.50$ & $2.68 \pm 0.51$ & \multirow{2}{*}{0.347} \\
& Range & $1.00-3.00$ & $1.00-3.00$ & \\
\multirow{2}{*}{ Retained embryos } & Mean $\pm \mathrm{SD}$ & $0.70 \pm 0.50$ & $0.74 \pm 0.44$ & $\wedge$ \\
& Range & $0.00-2.00$ & $0.00-1.00$ & \\
Transferred & Mean $\pm \mathrm{SD}$ & $1.93 \pm 0.74$ & $1.95 \pm 0.61$ & \\
embryos & Range & $1.00-3.00$ & $1.00-3.00$ & 0.787 \\
\multicolumn{2}{c}{ US visibility } & $144(52.9 \%)$ & $163(58.8 \%)$ & $\# 0.146$ \\
\hline
\end{tabular}

${ }^{\wedge}$ Independent t-test, \#Chi square test

Table 5 shows that no significant difference between Wallace and Prince (without mandrel) groups regarding pregnancy outcomes.

Table 5: Pregnancy outcomes among Wallace and Prince (without mandrel) groups

\begin{tabular}{lcccc}
\hline Findings & $\begin{array}{c}\text { Wallace } \\
(\mathrm{N}=300)\end{array}$ & $\begin{array}{c}\text { Prince } \\
(\mathrm{N}=300)\end{array}$ & $\mathrm{P}$ & RR (95\% CI) \\
\hline Biochemical pregnancy & $149(49.7 \%)$ & $157(52.3 \%)$ & $\# 0.514$ & $0.95(0.81-1.11)$ \\
Clinical pregnancy & $138(46.0 \%)$ & $145(48.3 \%)$ & $\# 0.567$ & $0.95(0.80-1.13)$ \\
Multiple pregnancy & $34(11.3 \%)$ & $38(12.7 \%)$ & $\# 0.615$ & $0.89(0.58-1.38)$ \\
Implantation rate & $33.0 \%$ & $34.5 \%$ & $\# 0.596$ & $0.96(0.81-1.13)$ \\
$(=$ Sacs/ transferred) & $173 / 525$ & $190 / 551$ & $\& 1.000$ & $1.00(0.20-4.91)$ \\
Miscarriage & $3(1.0 \%)$ & $3(1.0 \%)$ & $\&$
\end{tabular}

\#Chi square test, \&Fisher's Exact test, RR: Relative rate, CI: Confidence interval

\section{DISCUSSION}

Various case and embryonic factors affect the clinical outcome of ART protocols. The clinical tools for prediction of successful ART management cycle involve female age, ovarian reservoir, embryonic quality endometrial lining receptivity and embryonic transfer technique that is greatly influenced and affected by the type of catheter used. Embryonic transfer, the last step in management course of an ART cycle is considered a corner stone factor in ART success $^{[7]}$.

Variables affecting pregnancy rates following embryonic transfer involve sonographic guidance, easiness of embryonic transfer procedure, embryonic catheter type, transfer and catheter-loading procedure technique, blood or mucus impact, embryos retained, mock transfer performance, the reproductive clinician level of experience and catheter tip placement. In spite of the lack of harmony concerning the optimal technique of embryonic transfer, it is generally acclaimed that during embryonic transfer, the endometrial lining disruption and uterine contractions triggering should be avoided as much as possible. The affection of embryos by ambient conditions should be reduced and the embryo(s) should be placed at an optimal zone within the fundal area of the endometrial cavity ${ }^{[8]}$.

Various embryonic transfer catheters are commercially available for usage in reproductive medicine practice.
Interestingly, differences in catheter design involve stiff or soft materials, end or side openings and the existence of an outer sheath, malleability and quality of the implemented materials in manufacture. An acceptable catheter for human embryo transfer procedure is preferred by reproductive clinicians to be easy in usage and handling and have to be properly positioned within the uterus ${ }^{[9]}$.

Furthermore, it should be manufactured from nontoxic material and should be simple and economic. Stiff catheters and usage of a rigid outer sheath make catheter placement simpler but could cause more bleeding, trauma, mucus plugging and triggering of uterine contractility. Soft catheters permit the tip to follow the cervical contour and uterine accessible track and reduce endometrial lining trauma. The value of using of one embryonic transfer catheter over another is debatable and is under continuous research efforts ${ }^{[10]}$.

Initially the current research study enrolled 600 cases that were randomized into two research groups (Wallace number of cases $=300$, Prince without mandrel number of cases $=300$ ). From the total number of recruited study subjects, 31 cases were categorized as difficult transfer and no cases were lost in follow up. In basal characteristics among Wallace and Prince (without mandrel) research groups, there was no statistical significant difference between Wallace and Prince (without mandrel) research 
groups concerning basal characteristics of cases enrolled in the research study (age, BMI, FSH, LH, E2, Progesterone, etiology with $p$ values $=0.668,0.754,0.482,0.504,0.988$, $0.961,0.117$, consecutively).

Ovarian stimulation features and fertilization rates among Wallace and Prince (without mandrel) groups revealed that there was no statistical significant difference between Wallace and Prince (without mandrel) research groups as regards ovarian stimulation characteristics (total gonadotrophins, duration of stimulation, oocytes retrieved, oocytes injected fertilized oocytes, $\mathrm{p}$ values $=0.535$, $0.645,0.269,0.550,0.582,0.983,0.612$, consecutively), fertilization rates ( $\mathrm{p}$ value $=0.983$ ) and good embryonic quality $(\mathrm{p}$ value $=0.612$ ).

Furthermore, the current research study results revealed regarding transfer difficulty and patients' discomfort among Wallace and Prince (without mandrel) research groups that there was no statistical significant difference between Wallace and Prince (without mandrel) research groups regarding transfer difficulty and patients' discomfort ( $\mathrm{p}$ values $=0.456,0.254$, consecutively). There was no statistical significant difference between Wallace and Prince (without mandrel) research groups regarding transfer characteristics (loaded embryos, retained embryos transferred embryos, ultrasound visibility with $\mathrm{p}$ values $=0.347,0.393,0.787,0.146$, consecutively) .

Additionally, the current research has shown that there was no statistical significant difference between Wallace and Prince (without mandrel) research groups regarding pregnancy outcomes (biochemical pregnancy, clinical pregnancy, multiple pregnancy, implantation rates, miscarriages, live birth with $\mathrm{p}$ values $=0.514,0.567,0.615$, $0.596,1,0.566$, consecutively). No statistical significant difference existed between Labotech and Prince (with mandrel) research groups regarding basal characteristics (age, BMI, FSH, LH, E2, Progesterone, etiology of infertility with $p$ values $=0.217,0.304,0.077,0.083,0.143$, $0.889,0.825$, consecutively).

Furthermore in the current research results, there was no statistical significant difference between Labotech and Prince (with mandrel) research groups regarding ovarian stimulation characteristics (total gonadotrophins dosage, duration of ovarian stimulation, oocytes retrieved, oocytes injected fertilized oocytes with $\mathrm{p}$ values $=0.918,0.770$, $0.622,0.542,0.377$, consecutively), fertilization rates ( $p$ value $=0.608$ ) and embryonic good quality ( $p$ value $=0.202$ ). Interestingly, discomfort during embryonic transfer was observed statistically significantly more frequent in Labotech research group ( $p$ value $=0.016$ ). On the other hand, there was no significant difference between Labotech and Prince (with mandrel) research groups concerning embryo transfer features (loaded embryos, retained embryos, transferred embryos and ultrasound visibility with $\mathrm{p}$ values $=0.214,0.644,0.604,0.322$, consecutively)

Additionally in the current research results, there was no statistical significant difference between Labotech and Prince (with mandrel) research groups concerning pregnancy outcomes (biochemical pregnancy, clinical pregnancy, implantation rate miscarriage, live birth with $\mathrm{p}$ values $=.0 .683,0.688,0.610,0.317,1.00,0.691$, consecutively).

Interestingly, pregnant cases in the current research findings obtained have shown statistically significantly higher embryo good quality ( $p$ value $=0.012$ ), loaded embryos and transferred embryos as well as significantly lower retained embryos ( $p$ values $<0.001$ ).

A considerably prominent finding obtained from analysis of the current research results was that age and BMI were observed to be statistical significant factors that decrease live birth by their increase in Wallace research group ( $p$ values $<0.001$ ), in Prince without Mandrel research group ( $p$ values $=0.02,00.012$, consecutively), in Labotech research group ( $\mathrm{p}$ values $<0.001,0.008$, consecutively) in prince with Mandrel research group ( $\mathrm{p}$ values $=0.03$, $<0.001$, consecutively) while transferred embryos was a significant factor that increase live birth by its increase as observed in all research groups (Wallace, Prince without Mandrel, Labotech, prince with Mandrel having $p$ values $<0.001,0.015,0.045,<0.001$, consecutively).

A prior research study similar in methodology to the current research have revealed that the performance of soft catheters (Labotect, Wallace) was best in IVF-ET program that contradicts with the current study results in which there was no statistical difference observed. Furthermore, reproductive researchers observing differences between transfer catheters have shown that the Frydman catheter yielded the lowest rates of pregnancy ${ }^{[10]}$.

Differences in results could be justified by the fact that various issues such as the number of embryos transferred in the four research groups of cases in the previous research in comparison to the current research. To determine the statistical significance of number of embryos transferred upon rates of pregnancy prior research groups analyzed the correlation between the type of catheter and success rate, keeping the number of embryos fixed to one, two, three and four showing no statistical difference in rates of success rate with any of the catheters types that is in great harmony with the current research study findings ${ }^{[7]}$.

Furthermore, despite the fact that some research studies revealed no statistical significant difference in the performance of different embryonic transfer catheters in terms of pregnancy rates in harmony with the current research study findings, research studies have revealed statistically significantly better performance level of one embryo catheter over other in correlation to success rate $^{[3]}$.

Another research team performed a comparison between the (rigid) Erlangen catheter to the (soft) Wallace catheter in 320 cases in a randomized controlled research trial. The pregnancy rate per embryonic transfer was apparently higher in the Erlangen research group than in 
the Wallace research group, but the difference was not statistically significant ${ }^{[9]}$.

Additionally another reproductive research group compared the (soft) Wallace catheter to (rigid) TDT catheter in 428 cases having an embryo transfer revealing that both catheters performed similarly, even though there was a slight, but non-significant rise in rates of clinical pregnancy and implantation with Wallace catheter $(41.6 \%$ vs $36.0 \%$ and $16 \%$ vs $14.4 \%$ respectively). Correspondingly, AlShawaf research team of investigators revealed and displayed that there was no statistical difference in the reproductive performance of the (soft) Wallace and the (rigid) Frydman catheters concerning rates of pregnancy $(30.03 \% \text { vs } 30.7 \% \text { consecutively })^{[10]}$.

On the other hand, a prior research team of investigators performed a prospective randomized research study of 400 consecutive embryo transfers recommended the usage of the Frydman set over the Wallace catheter and TDT catheter due to higher rates of pregnancy $(32.3 \%$ vs $19.2 \%$ and $19.4 \%$, consecutively $)^{[8]}$.

Interestingly, another prospective randomized clinical research study, in which investigators compared 32 cases that had embryo transfer using the Tomcat catheter with 34 cases using the TDT catheter. The research team observed that the Tomcat catheter resulted in higher rates of implantation $(25.2 \%$ vs $8.4 \%)$ and rates of clinical pregnancy ( $47 \%$ vs $14.7 \%)$ in comparison with those with TDT catheter. Another research priorly conducted by reproductive researchers by performing statistical comparison of rates of clinical pregnancy in 518 cycles in cases undergoing embryonic transfer revealed that the rate of clinical pregnancy rate in women using soft catheters (Wallace and TDT) were statistically significantly higher than in those using hard catheters (Tomcat and Tefcat) $36 \%$ vs $17 \%$. Consecutively, all these mentioned studies contradict with the current research study results in which these differences were not observed.

Furthermore, a prior research review has come to the conclusion that flexible catheters have better rates of pregnancy in comparison to rigid catheters and Ruhlmann et al. ${ }^{[8]}$ research team of investigators has displayed that the rate of implantation rose when implementing flexible catheters rather than semi-rigid ones. Several research studies have compared soft catheters. As regards a prior comparison performed between Cook Soft and the Edwards-Wallace catheters, there was no statistically significant difference between them concerning rates of pregnancy ${ }^{[11]}$.

Additionally, another study comparing those two flexible embryonic catheters were conducted using a larger sample ( 75 cases for each research group) and they also found no statistical significant difference in pregnancy rates between the groups. On the contrary, another group of reproductive researchers comparing the same two types of embryonic catheters revealed that rates of pregnancy rates with the Cook's was 5\% higher than with the Wallace's:
$63.3 \%$ vs. $58.0 \%$, consecutively; but the research study power was not adequate to reveal statistically significant difference ${ }^{[7]}$

\section{CONCLUSION}

The current research study investigated four types of embryonic catheters Wallace, Prince with and without mandrel and Labotech most of the research study findings did not reveal any statistical significant differences by statistical analysis in which basic features and ovarian stimulation features did not differ between research groups categorized according to the type of catheter implemented.

However, considerably the cases age and BMI were the most prominent characteristics among the various research groups affecting live birth rates besides the transferred embryos. These findings denoted that endometrial, ovarian quality according to the age and BMI of cases could be the cornerstone factors in success of ICSI clinical outcomes more than the type of catheter implemented. However, other research variables should be respected that could cause difficult transfer such as anatomic challenges e.g cervical stenosis and submucous myomas that could affect catheter positioning and would reveal differences in the type of catheter used. The current research in addition raises a considerable issue of interest if BMI differences would affect the type and technique of embryo transfer by expected anatomical differences due to variability in body weight.

On the other hand, does the age of patient affect the genital tract anatomy in a manner even if trivial influencing the embryonic transfers process? Furthermore, do racial and ethnic differences affect the anatomical status of the cervix therefore impacting the transfer process? Sub clinical and clinical infections of the genital tract (e.g cervicitis, subclinical endometritis) are factors that should be put into consideration in future research.

Another issue that should be considered in future research is the integration of uterine volume, cervical position, uterine axis as factors affecting the choice of the type of catheter that could aid in proper performance of this meticulous art of embryonic transfer besides differences in experience and practice performed by the reproductive clinician should be put into consideration. Future research studies should consider multicentric fashion of research with great consideration of age, BMI, ethnicity, infections (causing cervical edema) as factors affecting the anatomic integrity and position; therefore, affecting the ease of the embryonic transfer process.

\section{CONFLICT OF INTEREST}

There are no conflicts of interest.

\section{REFERENCES}

1. Buckett WM (2006): A review and. meta-analysis of prospective ' trials comparing different catheters, used for embryo transfer. Fertility and Sterility; 85: 728-734. 
2. Meriano J, Weissman A, Greenblatt EM, et al. (2000): The choice of embryo transfer catheter affects embryo implantation after IVF. Fertility and Sterility; 74: 678-682.

3. Abou-Setta AM, Al-lnany HG, Mansour RT, et al. (2005): Soft versus firm embryo transfer catheters; for assisted reproduction; a systematic review andmeta-analysis. Human Reproduction; 20: 3114-3121 Wood EG, Batzer F, Go K, et al. (2000): Ultrasound guided soft catheter embryo transfers will improve pregnancy rates in in-vitro fertilization. Human Reproduction; 15: 107-112.

4. Hearns-Stokes RM, Miller BT, Scott L, et al. (2000): Pregnancy rates after embryo transfer depend on provider at embryo transfer, prospective randomized trial. Fertility and Sterility; 84: 996-1000.

5. Boone WR, Johnson JE, Blackhurst DM, et al. (2001): Cook versus Edwards-Wallace: are there differences in flexible catheters? Journal of Assisted.
Reproduction and Genetics; 18: 15-17.

6. Omidi M, Halvaei I, Mangoli E, et al. (2015): The effect of embryo catheter loading technique on the live birth rate. Clin Exp Reprod Med.; 42: 175-180.

7. Ruhlmann C, Gnocchi DC, Cattaneo AR, et al. (2015): Embryo Transfer Catheters: Softer is Easier. JBRA Assist Reprod.; 19: 204-249.

8. Tiras B and Cenksoy PO (2014): Practice of embryo transfer: recommendations during and after. In Seminars in Reproductive Medicine; 32(4): 291-296.

9. De Conto E, Schuster AK, Genro VK, et al. (2017): A prospective study comparing two embryotransfer soft catheters JBRA Assisted Reproduction; 21(2): 70-72.

10. Pierzyński $\mathrm{P}$ and Zbucka-Kretowska M (2014): Uterine contractile activity at embryo transferas a new pharmaco therapeutictarget in assisted reproduction. Ginekol Pol.; 85: 609-613. 